\title{
Evaluation of Allelopathic Effects of Iranian Rice Cultivars (Oryza sativa L.) on Growth Factors of Barnyard Grass (Echinochloa cruss-galli L.)
}

\author{
Seyed Ali Mohammad Modarres Sanavy ${ }^{1}$, Mohammadreza Delfieh ${ }^{1} \&$ Saeid Ghahary ${ }^{1}$ \\ ${ }^{1}$ Agronomy Dept., Faculty of Agriculture, Tarbiat Modares University, Tehran, Iran \\ Correspondence: Seyyed Ali Mohammad Modarres Sanavy, Agronomy Dept., Faculty of Agriculture, Tarbiat \\ Modares University, Tehran, Iran. Tel: 98-21-4829-2095. E-mail: modaresa@modares.ac.ir
}

Received: June 19, 2013 Accepted: October 12, 2014 Online Published: September 15, 2015

doi:10.5539/jas.v7n10p129 URL: http://dx.doi.org/10.5539/jas.v7n10p129

\begin{abstract}
In order to investigate the ability of aqueous hull extract of Iranian rice cultivars in controlling barnyard grass germination and seedlings growth, a factorial experiment was carried out at both Laboratory and greenhouse based on RCB (Randomized Complete Blocks) with 3 replication. In this study which was performed in 2010 at Tarbiat Modares University, the effects of 20 rice cultivars hull extract were considered on germination and seedling growth of barnyard grass weed at four concentrations $(0,5,10$ and 15 percent). Lab studies showed that some of the rice cultivars have stimulating effects on barnyard grass growth, while some others have inhibiting effects. Khazar cultivar showed the highest inhibiting effect on germination percentage, germination rate, radicle length, plumule length, radicle and plumule dry weight. Breeded cultivars had higher inhibition effects than natives, while the native cultivars were more stimulating on barnyard grass seed germination. The greenhouse part of study showed that Khazar cultivar causes significant reduction in germination percentage, germination rate, radicle length, radicle and plumule dry weight and whole plant dry weight of barnyard grass. Neda cultivar showed the highest effect in controlling plumule length. The study showed that various rice cultivars have different effects on inhibiting or stimulating the growth factors of barnyard grass.
\end{abstract}

Keywords: rice, hull, extract, barnyard grass, allelopathy

\section{Introduction}

The use of allelopathy could be an important step in the way of declining herbicide utilization, declining production expenses, environment conservation, sustainability of natural ecosystems and establishment of sustainable agriculture. Any procedure which is responsible of producing secondary metabolites in plants, microorganisms, viruses and fungi and affects the growth and development of agricultural and biological systems negatively or positively, could be called allelopathy (Torres et al., 1996). The chemicals which are responsible of allelopathy are called allelochemicals. The study of plant allelopathy was formalized in the 1980s, when Dilday discovered that a few rice cultivars had a special ability to inhibit the growth of paddy weeds, specifically red stem (Ammannia coccinea Rottb.) and duck salad (Heteranthera limosa (Sw.) Willd.) (Dilday et al., 1994).Allelopathy, properly applied, can reduce the need for chemical herbicides in rice cultivation, reducing the risk of environmental contamination, human health problems, and the development of herbicide-resistant weeds (Olofsdotter, 1998). The use of rice allelopathy in integrated weed management is one of the most interesting new avenues to sustainable agriculture (He et al., 2012).

Rice, after wheat, is the second largest cereal crop and the most widely consumed staple food grain. Globally, rice occupies about 145 million ha, a surface which constitutes one-tenth of the arable land, while in the majority of Asian countries, it comprises one-third or more of the planted area (Ferrero \& Tinarelli, 2008). Barnyard grass (Echinochloa crusgalli (L.) Beauv.), a C4 grass species morphologically similar to rice during the vegetative stage, competes with rice for nutrients, water, and other resources, reducing rice yield from 30 to $100 \%$ (Tang et al., 2009). There is no way other than biological control as the daily increasing utilization of herbicides has been led to increment in human diseases. Between 1993 to 1996 a thousand rice cultivars cultivated in Egypt with a $\mathrm{RCB}$ design and 3 replications in order to investigate the effect of them on barnyard grass. Forty days later, a significant decrement in the barnyard grass weight was observed. About 30 cultivars had 50 to 90 percent 
controlling effect on barnyard grass weed and more than 10 cultivars resulted in 50 to 70 percent weight loss in weed. In greenhouse experiments they observed 56 to 75 percent growth decrement in this weed. In this experiment weed growth and number of leaves showed a significant decrement. At the experiments which was carried out in Konkuk University of Korea and in three parts, lab, greenhouse and farm the effect of aqueous extract of rice shoot on the barnyard grass seed was investigated. Results showed that the extract of some cultivars had $61 \%$ growth inhibition on the weed and made $23 \%$ decrease in barnyard grass germination and $46 \%$ decrease in germination rate. At the greenhouse part there was the most inhibition with the rate of $73 \%$ decrement in dry weight, $74 \%$ decrement in shoot height and $57 \%$ decrement in germination percentage. At the farm part of experiments the most inhibition was observed in the number of tillers $(80 \%)$, leaf area $(49 \%)$, dry weight $(63 \%)$ and shoot height $(74 \%)$. This study recommended that the differences in rice inhibition on barnyard grass has a wide range (Chung et al., 2001). Based on the experiments which was carried out at Konkuk University of Korea in 2003 the allelopathical effects of rice on barnyard grass has been investigated and it was resulted that the extract of cultivars hull had about $76.9 \%$ inhibition effect on this weed. But the leaf and the epicarp extracts showed $74.1 \%$ and $31.7 \%$ inhibition respectively. The aggregate (extract of hull, leaf and epicarp) showed $38.6 \%$ inhibition. In overall it was observed that the extract of shoot show the best control in comparison to the various parts. In these experiments the relationship between the cultivars genetic and the allelopathical effects was compared. The native cultivars had $12.9 \%$ control and the introduced cultivars had $14.2 \%$ control on weeds. The inhibition rate in awn bearing cultivars was $16 \%$ and in awn less cultivars was $12 \%$ (Chung et al., 2003).

In road to achieve a sustainable agricultural system, it is so important for natural adjuvant compounds to be focused at. Hence considering the allelopathic potential of rice residues especially in its hull, and also assuming the intra-specific differences in this case, this study designed and carried out.

\section{Method}

Fully mature and awn bearing barnyard grass seeds were accumulated from a farm in Ghaemshahr. This weed is one of the most abundant weeds in farms and gardens (especially in paddy fields) of Iran and the rest of the world. After accumulation, seeds were put in $-20{ }^{\circ} \mathrm{C}$ in order the seed dormancy to be broken. After harvesting the rice yield and accumulation of paddy, a part of seeds were transferred to the paddy factory for detaching the hull. The hull was crunched with the mill. Then seed and hull were conserved in $5{ }^{\circ} \mathrm{C}$ until the experiment time. The 20 rice cultivars which were provided from the Amol Rice Research Centre were as follows: Behnam Chaloos, Tarom Chini, Sang Tarom, Nedaye Ramazani, Tarom Hashemi, Tarom Mahalli, Gerdeh, Kalat Dargaz, Ali Kazemi, Khazar, Fajr, Shafagh, Shiroudi, Tabesh, Nemat, Sahel, Kadous, Pouya, Zarak and Neda. The first nine cultivars are native and others are breed. Selection of these cultivars for this study was because of their broad utilization in north of Iran for both farming and research activities. Extraction was carried out based on the method of Ahn and Chung (2000). 30 gram of hull was put in $200 \mathrm{ml}$ distilled water and was shaken for an hour. The related extract was transferred through a 4 layer loose texture linen in order to filter the fiber residues. The produced solute was put in a low rounding centrifuge $(3000 \mathrm{rpm})$ for 4 hours. The produced matter was transferred from filter paper and then conserved in dark cap bearing bottles in fridge at $4{ }^{\circ} \mathrm{C}$. This solute which is called stock solute was the $15 \%$ concentration for this experiment. Two other concentrations of 5 and 10 percent are also been made. The experiment was carried out at two parts at lab and greenhouse. 
Table 1. Rice cultivars specifications

\begin{tabular}{lll}
\hline Cultivar Name & Subspecies & Specifications \\
\hline BehnamChaloos & Japonica & \\
TaromChini & Indica & Native species of North of Iran \\
SangTarom & Indica & Bred \\
NedayeRamazani & Indica & Selected from the native cultivars of Guilan province (North of Iran) \\
TaromHashemi & Indica & Native to Mazandaran province (North of Iran) \\
TaromMahalli & Indica & \\
Gerdeh & Japonica & \\
KalatDargaz & Indica & Selected from the native cultivars of Guilan province (North of Iran) \\
Ali Kazemi & Indica & Introduced cultivar (Rice research center of Rasht-Guilan province) \\
Khazar & Indica & Introduced cultivar (Amol research center-Mazandaran province) \\
Fajr & Indica & Introduced cultivar (Amol research center-Mazandaran province) \\
Shafagh & Indica & Introduced cultivar (Amol research center-Mazandaran province) \\
Shiroudi & Indica & Introduced cultivar (Amol research center-Mazandaran province) \\
Tabesh & Indica & Introduced cultivar (Amol research center-Mazandaran province) \\
Nemat & Indica & Introduced cultivar (Amol research center-Mazandaran province) \\
Sahel & Indica & Introduced cultivar (Rice research center of Rasht-Guilan province) \\
Kadous & Indica & Introduced cultivar (Amol research center-Mazandaran province) \\
Pouya & Indica & Naponica \\
Zarak & Indica & Bred cultivar (Amol research center-Mazandaran province) \\
Neda & &
\end{tabular}

\subsection{Lab Part}

Barnyard grass seeds became sterile with sodium hypochlorite solute at the volume relativity of $1: 10(\mathrm{v} / \mathrm{v})$ and for 10 minutes then were washed with distilled water for several times and afterwards were put on filter paper in order to be desiccated. In every cap bearing petri dishes 25 sterile seeds were put and $10 \mathrm{cc}$ of each solute concentration was added. Then the petri dishes were put in germinator at $30{ }^{\circ} \mathrm{C}$ and for 12 days. For holding humidity, the filter papers were removed after the 6th day, germinating seeds were washed with distilled water and after desiccation $10 \mathrm{cc}$ of related extracts were added again to every petri dish. This experiment was carried out as a factorial based on completely randomized design with 3 replications and at 4 concentrations (Control, 5, 10 and 15 extract concentrations).

\subsection{Greenhouse Part}

In every $15 \times 12$ undrained pot 500 gram of Mazanderan province sterile soil was poured and was put in green house at $28{ }^{\circ} \mathrm{C} .10$ barnyard grass seeds were put in top layer of each pot and the pots were preserved at low water level until the germination time. When the height of sprout reached to $2 \mathrm{~cm}$ the water level was increased and maintained up to $5 \mathrm{~mm}$ of shoot height. The provided extracts were added to pot water at the planting time $(10 \mathrm{cc})$. After 21 days the plants were pulled out and after washing were considered for related traits. No chemical fertilizers or herbicides were added to the pots. Analysis of variance and comparisons of means with Duncan multiple ranges were carried out with SAS software. Germination rate was calculated according to the formula proposed by Maguire (1962):

$$
\text { Rate of germination }=X_{1} / Y_{1}+\left(X_{2}-X_{1}\right) / Y_{2}+\ldots+\left(X_{n}-X_{n-1}\right) / Y_{n}
$$

Where, $X_{n}=$ Number of germinated seeds at $n^{\text {th }}$ count and $Y_{n}=$ Number of counts from planting to ${ }^{\text {th }}$ count. The rate of inhibition or stimulation of rice cultivars hull on the barnyard grass growth was calculated with the following formula:

$$
\text { Inhibition Rate }=(\text { Control plant }- \text { Treated plant }) / \text { Control plant } \times 100
$$




\section{Results and Discussion}

The results of analysis of variances have been brought in Tables 2 and 3. The table of treatments interactions means comparison (cultivar $\mathrm{x}$ concentration) showed that there was significant difference between various cultivars and various concentrations. The following traits were considered:

Table 2. The results of ANOVA for considered traits at lab conditions

\begin{tabular}{|c|c|c|c|c|c|c|c|c|c|}
\hline \multirow[b]{2}{*}{ Source of Variation } & \multirow[b]{2}{*}{ df } & \multicolumn{8}{|c|}{ Mean Squares } \\
\hline & & $\begin{array}{l}\text { Germination } \\
\text { percentage }\end{array}$ & $\begin{array}{l}\text { Germination } \\
\text { rate }\end{array}$ & $\begin{array}{l}\text { Radicle } \\
\text { length }\end{array}$ & $\begin{array}{l}\text { Plumule } \\
\text { length }\end{array}$ & $\begin{array}{l}\text { Radicle } \\
\text { DW }\end{array}$ & Plumule DW & $\begin{array}{l}\text { Ralativity of radicle } \\
\text { to plumule length }\end{array}$ & $\begin{array}{l}\text { Ralativity of radicle } \\
\text { to plumule DW }\end{array}$ \\
\hline Replication & 2 & $0.83^{\text {ns }}$ & $0.69^{\text {ns }}$ & $1.88^{*}$ & $4.498^{* *}$ & $4.498^{* *}$ & $0.00000002^{* *}$ & $0.0078^{\mathrm{ns}}$ & $0.0016^{\mathrm{ns}}$ \\
\hline Cultuivar (A) & 19 & $4864.8^{* *}$ & $2202.6^{* *}$ & $1332.86^{* *}$ & $2414.82^{* *}$ & $4.25^{* *}$ & $0.0000074^{* *}$ & $3.99^{* *}$ & $1.9^{* *}$ \\
\hline Concentration (B) & 3 & $16641.6^{* *}$ & $1822.0^{* *}$ & $15438.99^{* *}$ & $47835.29^{* *}$ & $5.915^{* *}$ & $0.0000015^{* *}$ & $0.0000013^{* *}$ & $6.65^{* *}$ \\
\hline$A * B$ & 57 & $1848.7^{* *}$ & $670.59^{* *}$ & $541.58^{* *}$ & $824.46^{* *}$ & $2.21^{* *}$ & $0.0000031^{* *}$ & $0.000079^{* *}$ & $1.21^{* *}$ \\
\hline Exp. Error & 158 & 0.4494 & 0.33034 & 0.4817 & 0.4245 & 1.008 & 0.0000001 & 0.0000001 & 0.028 \\
\hline
\end{tabular}

Note. n.s: Non-significant; ${ }^{* *}$ and *: significant at 0.01 and 0.05 probability levels, respectively.

Table 3. The results of ANOVA for considered traits at greenhouse conditions

\begin{tabular}{|c|c|c|c|c|c|c|c|c|c|}
\hline \multirow[b]{2}{*}{ Source of Variation } & \multirow[b]{2}{*}{ df } & \multicolumn{8}{|c|}{ Mean Squares } \\
\hline & & $\begin{array}{l}\text { Germination } \\
\text { percentage }\end{array}$ & $\begin{array}{l}\text { Germination } \\
\text { rate }\end{array}$ & $\begin{array}{l}\text { Radicle } \\
\text { length }\end{array}$ & $\begin{array}{l}\text { Plumule } \\
\text { length }\end{array}$ & $\begin{array}{l}\text { Radicle } \\
\text { DW }\end{array}$ & Plumule DW & $\begin{array}{l}\text { Ralativity of radicle } \\
\text { to plumule length }\end{array}$ & $\begin{array}{l}\text { Ralativity of radicle } \\
\text { to plumule DW }\end{array}$ \\
\hline Replication & 2 & $0.94^{\mathrm{ns}}$ & $0.086^{*}$ & $9.33^{*}$ & $1.3396^{*}$ & $0.0000017^{* *}$ & $0.0000003^{\mathrm{ns}}$ & $0.00067^{*}$ & $0.00039^{*}$ \\
\hline Cultuivar (A) & 19 & $4407.2^{* *}$ & $788.25^{* *}$ & $5971.29^{* *}$ & $4029.7^{* *}$ & $0.00073^{* *}$ & $0.094^{* *}$ & $10.79^{* *}$ & $0.9892^{* *}$ \\
\hline Concentration (B) & 3 & $1793.7^{* *}$ & $27.61^{* *}$ & $66080.5^{* *}$ & $58993.8^{* *}$ & $0.0047^{* *}$ & $0.0602^{* *}$ & $8.74^{* *}$ & $0.126^{* *}$ \\
\hline$A * B$ & 57 & $396.93^{* *}$ & $261.34^{* *}$ & $2348.4^{* *}$ & $1325.4^{* *}$ & $0.00041^{* *}$ & $0.0261^{* *}$ & $5.03^{* *}$ & $0.465^{* *}$ \\
\hline Exp. Error & 158 & 0.998 & 1.98 & 2.65 & 0.9603 & 1.99 & 2.33 & 0.955 & 2.77 \\
\hline
\end{tabular}

Note. n.s: Non-significant; ${ }^{* *}$ and $*$ : significant at 0.01 and 0.05 probability levels, respectively.

\subsection{Germination Percentage}

\subsubsection{Lab Part}

The highest rate of inhibition was showed by the cultivars Khazar and Tabesh and the lowest was showed by the cultivars Sang Tarom and BehnamChalous. Khazar cultivar showed high control on barnyard grass germination at every 3 extract concentrations. The cultivars of Khazar and Tabesh were both from breeded, awn bearing cultivars. The highest barnyard grass germination percentage was showed in the cultivar Sang Tarom hull. With increment of extract concentration from 5 to $15 \%$, the controlling rate of the cultivars Kalat Dargaz, Tarom Hashemi, Neda and Fajr were increased and that from of the cultivars of Pouya and Zarak were decreased (Table 4). 
Table 4. Inhibition (+) or stimulation (-) percentage of considered traits at lab conditions

\begin{tabular}{|c|c|c|c|c|c|c|c|c|c|c|}
\hline Cultivar & $\begin{array}{l}\% \text { Extract } \\
\text { concent }\end{array}$ & $\begin{array}{l}\text { Germ. } \\
\text { percent }\end{array}$ & $\begin{array}{l}\text { Germ. } \\
\text { rate }\end{array}$ & $\begin{array}{l}\text { Radicle } \\
\text { length }\end{array}$ & $\begin{array}{l}\text { Plumule } \\
\text { length }\end{array}$ & $\begin{array}{l}\text { Radicle } \\
\text { DW }\end{array}$ & $\begin{array}{l}\text { Plumule } \\
\text { DW }\end{array}$ & Total DW & $\begin{array}{l}\text { Ralativity of } \\
\text { radicle to } \\
\text { plumule length }\end{array}$ & $\begin{array}{l}\text { Ralativity of } \\
\text { radicle to } \\
\text { plumule DW }\end{array}$ \\
\hline \multirow{3}{*}{ TaromHashemi } & 5 & 28.33 & -2.7 & 34.52 & 50.64 & -50 & -6.82 & -99.04 & -57.97 & -776 \\
\hline & 10 & 45.67 & 12.76 & 24.54 & 52.35 & -66.67 & -13.64 & -91.37 & -90.92 & -517 \\
\hline & 15 & 48.33 & 5.41 & 9.18 & 66.88 & -66.67 & -38.64 & -107.62 & -242.7 & -135 \\
\hline \multirow{3}{*}{ Khazar } & 5 & 100 & 48.5 & 55.13 & 85.8 & 0 & 0 & -119.04 & 0.64 & 0.13 \\
\hline & 10 & 100 & 48.5 & 55.13 & 85.8 & 0 & 0 & -123.81 & 0.64 & 0.13 \\
\hline & 15 & 100 & 48.5 & 55.13 & 85.8 & 0 & 0 & -129.52 & 0.64 & 0.13 \\
\hline \multirow{3}{*}{ TaromMahalli } & 5 & 81.33 & 37.57 & 43.65 & 74.92 & -50 & -20.45 & -114.28 & -101.5 & -258 \\
\hline & 10 & 0 & -51.2 & -30.72 & -1.07 & -150 & -6.82 & -87.62 & -100.1 & -2327 \\
\hline & 15 & 23 & -49.7 & -11.19 & 21.31 & -50 & -120.45 & -109.52 & -103.5 & -43.8 \\
\hline \multirow{3}{*}{ Shiroudi } & 5 & 7.33 & -48.7 & 37.54 & 50.33 & -66.67 & -20.45 & -71.43 & -48.98 & -345 \\
\hline & 10 & 0.67 & -76 & 46.55 & 44.43 & -50 & -52.27 & -66.67 & -20.1 & -101 \\
\hline & 15 & 0.67 & -76 & 46.55 & 44.43 & -50 & -52.27 & -103.88 & -20.1 & -101 \\
\hline \multirow{3}{*}{ Ali Kazemi } & 5 & 18 & 3 & 18.74 & 49.55 & -66.67 & -13.64 & -106.67 & -99.92 & -517 \\
\hline & 10 & 70.67 & -15.4 & 9.18 & 79.58 & -150 & -6.82 & -95.23 & -782.8 & -2327 \\
\hline & 15 & 2.33 & -81.1 & 14.14 & 76.86 & -50 & -6.82 & -67.62 & -440.5 & -776 \\
\hline \multirow{3}{*}{ Tabesh } & 5 & 75.67 & 37.5 & 50.90 & 76.28 & -50 & -13.64 & -97.15 & -45.51 & -388 \\
\hline & 10 & 66.67 & 29.81 & 44.92 & 58.61 & -66.67 & -34.09 & -96.19 & -35.21 & -207 \\
\hline & 15 & 85.67 & 41.97 & 50.90 & 80.36 & -50 & -13.64 & -105.71 & -67.31 & -411 \\
\hline \multirow{3}{*}{ Neda } & 5 & 0 & -70.7 & -0.49 & 79.58 & -83.33 & -6.82 & -93.33 & -858.4 & -1293 \\
\hline & 10 & 83.33 & 34.07 & 48.42 & 83.47 & -66.67 & -6.82 & -91.43 & -266.5 & -1034 \\
\hline & 15 & 100 & 48.5 & 55.13 & 85.8 & 0 & 0 & -92.37 & 0.64 & 0.13 \\
\hline \multirow{3}{*}{ TaromChini } & 5 & 0.33 & -66.7 & -68.39 & 42.29 & -150 & -6.82 & -85.71 & -283.3 & -2715 \\
\hline & 10 & 0 & -82.1 & -29.15 & 51.96 & -166.67 & -13.64 & -86.66 & -249.6 & -1293 \\
\hline & 15 & 0.67 & -80.7 & -11.07 & 44.7 & -150 & -54.54 & -101.9 & -160.5 & -291 \\
\hline \multirow{3}{*}{ BehnamChalous } & 5 & 0 & -53.6 & -45.17 & 28.11 & -150 & -31.82 & -78.09 & -180.5 & -468 \\
\hline & 10 & 0.67 & -74.2 & -15.24 & 64.04 & -100 & -13.64 & -42.85 & -318.9 & -776 \\
\hline & 15 & 0 & -80.9 & 21.1 & 34.13 & -66.67 & -34.1 & -86.66 & -65.24 & -207 \\
\hline \multirow{3}{*}{ Sang Tarom } & 5 & 0 & -80.4 & -62.16 & 34.13 & -166.67 & -47.73 & -81.9 & -228.3 & -335 \\
\hline & 10 & 0.33 & -79.1 & -33.5 & 22.2 & -100 & -34.1 & -63.81 & -144.6 & -310 \\
\hline & 15 & 0 & -81.3 & -14.52 & 17.23 & -100 & -59.1 & -85.71 & -101 & -179 \\
\hline \multirow{3}{*}{ Gerdeh } & 5 & 6.67 & -68.2 & -9.74 & 68.71 & -128.57 & -34.1 & -84.76 & -358.9 & -465 \\
\hline & 10 & 3.67 & -65.9 & 27.75 & 78.42 & -71.43 & -6.82 & -78.09 & -396.2 & -1293 \\
\hline & 15 & 100 & 48.5 & 55.13 & 85.8 & 0 & 0 & -98.09 & 0.64 & 0.13 \\
\hline \multirow{3}{*}{ KalatDargaz } & 5 & 0 & -77.9 & 13.42 & 70.69 & -83.33 & -22.73 & -72.38 & -271.2 & -429 \\
\hline & 10 & 0.67 & -49.1 & 38.63 & 79.74 & -66.67 & -6.82 & -93.33 & -304.1 & -1034 \\
\hline & 15 & 4.33 & -44.3 & 42.5 & 82.3 & -100 & -6.82 & -81.85 & -360.7 & -1552 \\
\hline \multirow{3}{*}{ Shafagh } & 5 & 0 & -81.3 & -45.83 & 21.5 & -171.43 & -47.73 & -84.86 & -158.6 & -443 \\
\hline & 10 & 26.67 & -31.2 & 41.11 & 77.29 & -85.71 & -31.82 & -70.04 & -164.2 & -313 \\
\hline & 15 & 13 & -50.3 & 49.33 & 80.36 & -42.86 & -13.64 & -60.95 & -117.1 & -396 \\
\hline \multirow{4}{*}{ Fajr } & 5 & 0 & -50.3 & -25.58 & 50.37 & -266.67 & -13.64 & -84.96 & -235.4 & -2069 \\
\hline & 10 & 0.33 & -78 & -6.23 & 71.23 & -50 & -6.82 & -83.81 & -434 & -776 \\
\hline & 15 & 36 & -22.6 & 45.4 & 82.27 & -66.67 & -13.64 & -78.09 & -282.3 & -517 \\
\hline & 5 & 8.67 & -65.6 & -40.82 & 20.07 & -250 & -81.82 & -90.47 & -145.7 & -323 \\
\hline NedaRamezani & 10 & 0.67 & -81.2 & -18.99 & 23.56 & -150 & -45.45 & -86.66 & -116.2 & -351 \\
\hline
\end{tabular}




\begin{tabular}{|c|c|c|c|c|c|c|c|c|c|c|}
\hline & 15 & 6.33 & -81.3 & 10.76 & 71.31 & -100 & -11.36 & -87.62 & -303.2 & -1034 \\
\hline \multirow{3}{*}{ Zarak } & 5 & 67.33 & 21.56 & 34.28 & 72.59 & -50 & -27.27 & -150.47 & -159.3 & -194 \\
\hline & 10 & 45 & -3.39 & 6.77 & 49.98 & -100 & -61.36 & -107.62 & -134.4 & -172 \\
\hline & 15 & 24.67 & 13.17 & 33.73 & 44.1 & -116.67 & -68.18 & -142.65 & -51.77 & -181 \\
\hline \multirow{3}{*}{ Pouya } & 5 & 65.67 & .25 .2 & 14.81 & 57.13 & -116.67 & -34.09 & -70.48 & -136 & -318 \\
\hline & 10 & 33.67 & -28.5 & 16.86 & 12.65 & -100 & -93.18 & -98.09 & -51.7 & -114 \\
\hline & 15 & 11.67 & -36 & -40.51 & -5.42 & -166.67 & -125 & -119.04 & -104.2 & -141 \\
\hline \multirow{3}{*}{ Kadous } & 5 & 56.33 & -3.6 & 13.78 & 44.58 & -66.67 & -45.45 & -142.85 & -99.68 & -109 \\
\hline & 10 & 37 & -31.8 & -33.08 & 13.66 & -50 & -54.54 & -106.66 & -121.6 & -96.9 \\
\hline & 15 & 59.33 & -7.38 & 10.82 & 36.03 & -50 & -56.82 & -100.95 & -85.84 & -97.1 \\
\hline \multirow{3}{*}{ Nemat } & 5 & 59.33 & 11.94 & 26.05 & 50.14 & -50 & -36.36 & -106.66 & -80.88 & -152 \\
\hline & 10 & 24.67 & -33.4 & 2.96 & 14.7 & -66.67 & -34.1 & -107.62 & -72.75 & -160 \\
\hline & 15 & 45.33 & -18.9 & -10.71 & -5.3 & -116.67 & -54.54 & -107.62 & -71.63 & -189 \\
\hline \multirow{3}{*}{ Sahel } & 5 & 39.33 & -15.1 & 11.48 & 33.94 & -100 & -75 & -118.09 & -83.52 & -141 \\
\hline & 10 & 46.33 & -6.62 & 28.71 & 22.36 & -116.67 & -84.09 & -133.33 & -42.4 & -128 \\
\hline & 15 & 24 & -60.6 & -2.49 & 8.57 & -100 & -54.54 & -92.28 & -73.96 & -196 \\
\hline
\end{tabular}

\subsubsection{Greenhouse Part}

The highest rate of inhibition was showed by the cultivars of Khazar and Tabesh. These were breeded cultivars. The cultivars of BehnamChalous, TaromChini and Sang Tarom showed the lowest inhibition on barnyard grass germination percentage. With increment of extract concentration from 5 to $15 \%$, the controlling rate of the cultivars Fajr, Neda and BehnamChalous increased where this trait decreased in the cultivars Zarak and Pouya (Table 5). In overall, the native cultivars had the lowest inhibition on barnyard grass weed and acted as a stimulator of barnyard grass germination (Table 5). Kim and Shin (1996) reported that rice hull inhibits barnyard grass germination with the rate of $70 \%$. Hassan et al. (1995) reported 50 to $90 \%$ inhibition. Ahn and Chung (2000) showed 75 to $95 \%$ as inhibition ability of rice hull extract on barnyard grass weed germination.

Table 5. Inhibition $(+)$ or stimulation $(-)$ percentage of considered traits at greenhouse conditions

\begin{tabular}{|c|c|c|c|c|c|c|c|c|c|c|}
\hline Cultivar & $\begin{array}{l}\% \\
\text { Extract } \\
\text { concent }\end{array}$ & $\begin{array}{l}\text { Germ. } \\
\text { percent }\end{array}$ & $\begin{array}{l}\text { Germ. } \\
\text { rate }\end{array}$ & $\begin{array}{l}\text { Radicle } \\
\text { length }\end{array}$ & $\begin{array}{l}\text { Plumule } \\
\text { length }\end{array}$ & $\begin{array}{l}\text { Radicle } \\
\text { DW }\end{array}$ & $\begin{array}{l}\text { Plumule } \\
\text { DW }\end{array}$ & $\begin{array}{l}\text { Total } \\
\text { DW }\end{array}$ & $\begin{array}{l}\text { Ralativity of } \\
\text { radicle to } \\
\text { plumule length }\end{array}$ & $\begin{array}{l}\text { Ralativity of } \\
\text { radicle to } \\
\text { plumule DW }\end{array}$ \\
\hline \multirow{3}{*}{ TaromHashemi } & 5 & 25.7 & -26.38 & 98.29 & 64.29 & -33.56 & -29.23 & 37.05 & -50.9 & -114 \\
\hline & 10 & 48.2 & -45.85 & 90.24 & 65.88 & -35.16 & -102.7 & 36.18 & -75.3 & -33.7 \\
\hline & 15 & 46.8 & -33.22 & 73.25 & 83.7 & -35.16 & -146.5 & 4.85 & -242 & -23.5 \\
\hline \multirow{3}{*}{ Khazar } & 5 & 84.5 & 5.86 & 105.85 & 85.46 & -29.56 & -96.3 & 51.46 & -71.5 & -30.2 \\
\hline & 10 & 79.2 & 4.28 & 107.66 & 94.58 & -27.16 & -136.5 & 58.75 & -138 & -19.4 \\
\hline & 15 & 94.7 & 25.33 & 118.1 & 102.33 & 0.04 & 0.08 & 94.9 & 1.15 & 0.5 \\
\hline \multirow{3}{*}{ TaromMahalli } & 5 & 81.6 & -6.9 & 107.09 & 89.83 & -32.76 & -118.4 & 5.97 & -88.1 & -27.2 \\
\hline & 10 & -2.5 & -88.48 & 34.53 & 11.65 & -116 & -29.23 & 24.23 & -91 & -403 \\
\hline & 15 & -9.2 & -68.88 & 54.85 & 28.33 & -102.4 & -55.34 & 44.13 & -83.3 & -184 \\
\hline \multirow{3}{*}{ Shiroudi } & 5 & 9.46 & -82.69 & 101.25 & 67.48 & -33.56 & -57.35 & 60.43 & -46.1 & -58 \\
\hline & 10 & -1.1 & -136.5 & 109.12 & 52.53 & -28.76 & -256.5 & 7.27 & -16.9 & -10.7 \\
\hline & 15 & 68.6 & -96.38 & 105.96 & 91.45 & -32.76 & -105.9 & 34.12 & -113 & -30.4 \\
\hline \multirow{3}{*}{ Ali Kazemi } & 5 & 16.1 & -93.09 & 83.58 & 91.98 & -32.76 & -62.97 & 13.36 & -325 & -51.5 \\
\hline & 10 & 73.2 & -24.93 & 73.99 & 72.82 & -108.8 & -28.83 & 33.98 & -150 & -371 \\
\hline & 15 & -2.5 & -144.9 & 84.23 & 95.1 & -60.76 & -28.03 & 65.42 & -472 & -211 \\
\hline \multirow{3}{*}{ Tabesh } & 5 & 77.1 & -17.17 & 116.04 & 94.35 & -29.56 & -58.15 & 54.35 & -24.6 & -50.3 \\
\hline & 10 & 62.6 & -25.19 & 107.43 & 70.87 & -33.56 & -172.6 & 64.98 & -32.8 & -19.4 \\
\hline & 15 & 83.8 & -17.04 & 113.98 & 97.61 & -35.16 & -64.17 & 61.64 & -88.5 & -54.3 \\
\hline
\end{tabular}




\begin{tabular}{|c|c|c|c|c|c|c|c|c|c|c|}
\hline & 5 & -5.3 & -120.9 & 62.78 & 96.31 & -60.76 & -32.85 & 67.07 & -963 & -184 \\
\hline \multirow[t]{3}{*}{ Neda } & 10 & 79.9 & 6.25 & 112.71 & 99.73 & -66.36 & -28.43 & 31.27 & -234 & -238 \\
\hline & 15 & 89 & 3.23 & 110.85 & 98.82 & -27.16 & -13.57 & 22.31 & -218 & -199 \\
\hline & 5 & -2.2 & -116.9 & 8.87 & 52.53 & -136 & -29.64 & 23.67 & -234 & -458 \\
\hline \multirow[t]{3}{*}{ TaromChini } & 10 & -2.5 & -148.4 & 24.54 & 68.16 & -102.4 & -66.58 & 39.9 & -277 & -153 \\
\hline & 15 & -2.2 & -145.7 & 48.44 & 53.73 & -51.16 & -52.93 & 11.79 & -142 & -96.1 \\
\hline & 5 & -4.3 & -105.1 & 13.3 & 39.34 & -100.8 & -152.5 & 12.47 & -164 & -67.9 \\
\hline \multirow[t]{3}{*}{ BehnamChalous } & 10 & -3.2 & -144.8 & 37.27 & 78.2 & -63.96 & -135.3 & 42.5 & -332 & -46.8 \\
\hline & 15 & 5.23 & -149.1 & 84.82 & 48.75 & -32.76 & -168.6 & 13.81 & -60.3 & -20.1 \\
\hline & 5 & 5.23 & -143.5 & 0.23 & 44 & -134.4 & -201.5 & 66.14 & -200 & -66.2 \\
\hline \multirow[t]{3}{*}{ Sang Tarom } & 10 & -0.4 & -139.5 & 33.43 & 30.57 & -61.65 & -172.6 & 50.34 & -117 & -35.2 \\
\hline & 15 & 6.29 & -141.2 & 42.99 & 20.09 & -50.36 & -293.1 & 14.53 & -90.2 & -16.7 \\
\hline & 5 & 4.17 & -117.4 & 58.15 & 81.94 & -67.16 & -253.3 & 7.58 & -297 & -26 \\
\hline \multirow[t]{3}{*}{ Gerdeh } & 10 & -0.1 & -126 & 90.05 & 94.22 & -56.76 & -256.9 & 40.26 & -324 & -21.6 \\
\hline & 15 & 87.6 & -17.17 & 99.22 & 90.9 & -27.96 & -13.57 & 51.75 & -155 & -233 \\
\hline & 5 & 4.53 & -128 & 80.03 & 86.83 & -66.36 & -136.1 & 34.45 & -239 & -48.3 \\
\hline \multirow[t]{3}{*}{ KalatDargaz } & 10 & 1 & -67.96 & 98.74 & 96.14 & -31.96 & -29.23 & 45.75 & -312 & -113 \\
\hline & 15 & 2.77 & -74.67 & 107.52 & 98.29 & -63.96 & -34.86 & 72.31 & -302 & -179 \\
\hline & 5 & -0.8 & -131.8 & 30.04 & 24.81 & -133.6 & -257.3 & 11.79 & -112 & -51.4 \\
\hline \multirow[t]{3}{*}{ Shafagh } & 10 & 20.4 & -59.4 & 105.48 & 95.1 & -29.56 & -136.1 & 12.91 & -169 & -21.2 \\
\hline & 15 & 9.81 & -104.1 & 112.48 & 95.17 & -28.76 & -64.17 & 68.74 & -79.8 & -44.3 \\
\hline & 5 & -3.2 & -91.64 & 43.7 & 63.31 & -136.8 & -66.58 & 65.72 & -187 & -208 \\
\hline \multirow[t]{3}{*}{ Fajr } & 10 & 4.53 & -99.8 & 62.3 & 81.91 & -31.96 & -33.65 & 60.42 & -274 & -92.4 \\
\hline & 15 & 13.3 & -22.82 & 107.6 & 97.74 & -58.36 & -60.56 & 65.74 & -246 & -96.8 \\
\hline & 5 & 5.58 & -96.25 & 22.96 & 26.54 & -170.4 & -401.5 & 33.06 & -124 & -41.9 \\
\hline \multirow[t]{3}{*}{ NedaRamezani } & 10 & -1.1 & -135.9 & 46.69 & 39.14 & -91.96 & -134.5 & 54.4 & -112 & -67.9 \\
\hline & 15 & 9.81 & -138.2 & 77.37 & 87.28 & -107.2 & -57.35 & 57.83 & -269 & -185 \\
\hline & 5 & 71.1 & -5.06 & 88.13 & 74.55 & -33.56 & -156.5 & -8.85 & -107 & -20.9 \\
\hline \multirow[t]{3}{*}{ Zarak } & 10 & 47.5 & -54.27 & 65.88 & 48 & -76.76 & -349.3 & 38.55 & -95.3 & -21.4 \\
\hline & 15 & 30.6 & -42.3 & 95.69 & 42 & -59.16 & -437.7 & 45.73 & -36.1 & -13 \\
\hline & 5 & 80.9 & -75.72 & 77.57 & 60 & -78.36 & -208.8 & -8.85 & -97.2 & -37 \\
\hline \multirow[t]{3}{*}{ Pouya } & 10 & 39 & -96.25 & 84.85 & 21.42 & -65.56 & -538.1 & 35.55 & -40.1 & -11.7 \\
\hline & 15 & 18.3 & -137.6 & 14.83 & 9.79 & -97.56 & -767 & 45.43 & -111 & -12.2 \\
\hline & 5 & 78.1 & -27.69 & 74.55 & 47.94 & -27.16 & -381.4 & 69.66 & -79.2 & -6.61 \\
\hline \multirow[t]{3}{*}{ Kadous } & 10 & 48.2 & -71.77 & 43.64 & 21.13 & -39.96 & -265 & 38.46 & -90.9 & -14.5 \\
\hline & 15 & 79.2 & -55.32 & 65 & 39.43 & -51.96 & -321.2 & 22.6 & -83.6 & -15.6 \\
\hline & 5 & 72.8 & 7.31 & 89.96 & 51.88 & -32.76 & -172.6 & 5.32 & -54.8 & -18.4 \\
\hline \multirow[t]{3}{*}{ Nemat } & 10 & 27.1 & -65.19 & 56.66 & 30.8 & -44.76 & -216.8 & 45.34 & -85.1 & -20.1 \\
\hline & 15 & 74.2 & -52.82 & 62.13 & 11.71 & -60.76 & -329.2 & 59.46 & -60.8 & -17.9 \\
\hline & 5 & 55.2 & -55.06 & 56.71 & 37.87 & -60.76 & -510 & 32.68 & -94.4 & -11.4 \\
\hline \multirow[t]{2}{*}{ Sahel } & 10 & 38 & -35.98 & 85.59 & 42.86 & -31.16 & -610.4 & 43.66 & -53.7 & -4.59 \\
\hline & 15 & 14.4 & -104 & 18.72 & 18.98 & -78.36 & -397.5 & 41.24 & -119 & -19.2 \\
\hline
\end{tabular}

\subsection{Germination Rate}

\subsubsection{Lab Part}

The highest rate of inhibition was showed by the cultivar Khazar and at every 3 concentrations. The highest rate of stimulation was showed by the cultivars TaromChini, Sang Tarom and NedaRamezani. Three of them were from the native, awn bearing cultivars. With increment of extract concentration from 5 to $15 \%$, the controlling 
rate of the cultivars Neda, Gerdeh and KalatDargaz increased and that from the cultivar of Ali Kazemi, BehnamChalous, NedaRamazani and Pouya decreased (Table 4).

\subsubsection{Greenhouse Part}

The stimulation rate of the cultivars Sang Tarom, TaromChini and BehnamChalous was the highest. The highest rate of stimulation was showed by the cultivar BehnamChalous and at the $10 \%$ concentration. The cultivars Sang Tarom and TaromChini were native cultivars. With increment of extract concentration from 5 to $15 \%$, the stimulation rate of the cultivars NedaRamezani, BehnamChalous and Pouya increased (Table 5).

In overall, inhibition on the germination rate of breeded cultivars was higher. Chung et al. (2001) resulted that the inhibition rate of rice extract on barnyard grass germination rate was 7 to 46 percent.

\subsection{Radicle Length}

\subsubsection{Lab Part}

Khazar cultivar showed the highest inhibition at every concentration and the cultivar of Sang Tarom showed the highest stimulation. With increment of extract concentration from 5 to $15 \%$, the inhibition rate of the cultivars Neda, NedaRamazani, Gerdeh, KalatDargaz, BehnamChalous and Shiroudi increased and that of the cultivars of Nemat and TaromHashemi decreased (Table 4).

\subsubsection{Greenhouse Part}

The highest inhibition was showed by Khazar cultivar at $15 \%$ concentration. With increment of extract concentration from 5 to $15 \%$, the inhibition rate of the cultivars Khazar, Tarom Chini, Behnam Chalous, Gerdeh, Kalat Dargaz, Shafagh, Neda Ramazani and Fajr increased and that of the cultivar TaromHashemi decreased. In overall, the breeded cultivars had the highest inhibition while the native cultivars had the lowest inhibition on radicle length of barnyard grass weed. Olofsdotter and Navarez (1996) resulted that rice has significant inhibition effect on the radicle length of barnyard grass weed. Hassn et al. (1994) reported that some rice cultivars reduce root progress of barnyard grass weed significantly.

\subsection{Plumule Length}

\subsubsection{Lab Part}

Khazar cultivar at its 3 concentrations and then the cultivars of Neda and Gerdeh showed a very successful control effect. Three of them are from breeded, awn bearing cultivars. With increment of extract concentration the inhibition rate of the cultivars Neda, Tarom Hashemi, Gerdeh, Kalat Dargaz, Shafagh, Fajr and NedaRamazani increased and the cultivars Pouya, Sang Tarom, Zarak, Sahel and Nemat showed a significant decrease (Table 4).

\subsubsection{Greenhouse Part}

The highest inhibition rate was observed in the cultivars Khazar and Neda. With increment of extract concentration the inhibition rate of the cultivars Khazar, KalatDargaz, NedaRamazani, Shafagh, Fajr and TaromHashemi increased and that of the cultivars of Sang Tarom, Pouya, Zarak and Nemat decreased (Table 5). Asghari and Mousavi (2001) concluded that rice allelopathy causes a 31 to 55 percent reduce in barnyard grass weed plumule length. Asghari et al. (2006) concluded that Ali Kazemi cultivar had stmulating effect on shoot lenght.

\subsection{Radicle Dry Weight}

\subsubsection{Lab Part}

The cultivar of Khazar showed the highest inhibition effect while the cultivars of NedaRamezani and TaromChini showed the highest stimulation. With increment of extract concentration, the stimulation rate of the cultivars Zarak and Nemat increased. It means that these cultivars stimultae the barnyard grass weed to produce bulkier root (Table 4).

\subsubsection{Greenhouse Part}

The highest stimulation rate was observed in NedaRamezani cultivar at $5 \%$ concentration and the in the TaromChini cultivar. With increment of extract concentration from 5 to $15 \%$, the stimulation rate of the cultivars Kadous, Nemat and Tabesh increased. In overall, the native cultivars stimulated the barnyard grass weed to produce bulkier roots (Table 5). 
Chung et al. (2001) concluded that rice hull extract show significant inhibition on the barnyard grass weed radicle dry weight. Also the studies of Asghari et al. (2006) showed that Neda cultivar had more than $40 \%$ inhibitory effect on root dry weight.

\subsection{Plumule Dry Weight}

\subsubsection{Lab Part}

The highest inhibition rate was showed by Khazar cultivar at its three concentrations. The highest stimulation was showed by the cultivars Pouya and Sahel. With increment of extract concentration from 5 to $15 \%$, the inhibition rate of the cultivar Neda Ramezani, Shafagh and Gardeh and the stimulating rate of the cultivars Tarom Hashemi, Tarom Chini, Pouya, Zarak and Kadous increased (Table 4).

\subsubsection{Greenhouse Part}

Pouya cultivar at $15 \%$ concentration and Sahel cultivar showed the highest stimulation percentage. These two cultivars were breeded and awn less. With increment of extract concentration from 5 to $15 \%$, the stimulation rate of the cultivars Zarak, Pouya, Nemat and Tarom Hashemi increased (Table 5).

Chung et al. (2001) showed that the plumule dry weight of barnyard grass weed could be well controlled by the rice hull extract. In another research, Neda cultivar showed a high inhibitory effect on shoot dry weight (Asghari et al., 2006).

\subsection{Total Dry Weight}

\subsubsection{Lab Part}

Zarak cultivar showed the highest stimulation. The highest stimulation rate was observed from $5 \%$ concentration of Zarak cultivar. With increment of extract concentration from 5 to $15 \%$, the stimulation rate of the cultivars Pouya and TaromChini increased (Table 4).

\subsubsection{Greenhouse Part}

High inhibition rate was observed in Khazar and Fajr cultivars. Both are from breeded, awn bearing and short season cultivars. With increment of extract concentration the inhibition rate of the cultivars Gerdeh, Kalat Dargaz, Nemat, Khazar, Tarom Mahalli, Ali Kazemi, Shafagh, Neda Ramazani, Zarak and Pouya increased. In overall, the breeded cultivars showed higher inhibition rate on this trait (Table 5). Decrement of barnyard grass total dry weight has been reported by Hassan et al. (1995). Chung et al. (2001) reported that rice hull extract showed $73 \%$ decline in barnyard grass weed total dry weight. Xun et al. (2006) resulted that rice allelopathical ability for declination of barnyard grass weed total dry weight is $70 \%$.

\subsection{Relativity of Radicle Length to Plumule Length}

\subsubsection{Lab Part}

The highest rate of stimulation was observed in Ali Kazemi cultivar and the highest rate of inhibition was observed in Khazar cultivar. With increment of extract concentration from 5 to $15 \%$, the stimulation rate of the cultivars TaromHashemi and Kalatdargaz increased (Table 4).

\subsubsection{Greenhouse Part}

The highest stimulation was observed in the cultivars Neda and Kalat Dargaz. With increment of extract concentration from 5 to $15 \%$, the stimulation rate of TaromHashemi and Tabesh cultivars increased (Table 5). Olofsdotter and Navarez (1996) reported that the inhibition rate of rice hull extract on barnyard grass weed shoot is less that root.

\subsection{Relativity of Radicle to Plumule Dry Weight}

\subsubsection{Lab Part}

Khazar cultivar controlled radicle and plumule completely. Other cultivars caused stimulation i.e. caused increment in radicle length more than plumule length. Increment of extract concentration from 5 to $15 \%$, caused stimulation of the cultivars Nemat, Neda Ramezani and Kalat Dargaz to produce bulkier roots (Table 4).

\subsubsection{Greenhouse Part}

$5 \%$ concentration of TaromChini cultivar showed the highest stimulation where the $15 \%$ concentration of Khazar cultivar showed the highest inhibition. Sahel cultivar showed the lowest stimulation on barnyard grass weed growth. Increment of extract concentration from 5 to $15 \%$, caused stimulation of the cultivars Kalat Dargaz, Neda Ramezani and Kadous to produce bulkier roots. These cultivars were from native and awn bearing 
cultivars. Native cultivars made a powerful stimulation on barnyard grass weed growth (Increment in length and volume) (Table 5). Chung et al. (2001) resulted that barnyard grass root growth declination in relation to plumule is because of the usage of more rice hull which cause more contact of extract to plant roots. Similar report was presented by Kim et al. (2004).

In overall the results of this study showed that the hull extract of different rice cultivars could have significant inhibiting or stimulating effects on barnyard grass weed growth characteristics especially at the early growth stages. The inhibitory specification of some rice cultivars could be a key factor in formulating some new bioherbicides. The cultivars Khazar, Zarak, Kadous and Sahel showed a great potential in this case. It also may be possible to use rice straw and hull as a covering mulch in order to control the barnyard grass weed in conservation agriculture, however, achieving such goals needs more research activities.

\section{References}

Ahn, J. K., \& Change, I. M. (2000). Allelopathic potential of rice hull germination and seedling rowthof barnyardgrass. Agronomy Journal, 92, 1162-1167. http://dx.doi.org/10.2134/agronj2000.9261162x

Asghari, J., Berendji, S., Fotohi, H., Matin, A., \& Mohammad-Sharifi, M. (2006). Potential Allelopathic Effects of Rice Hull Extracts on Barnyardgrass (Echinochloa crus-galli) Seedling Growth. Iranian Journal of Weed Science, 2(2), 31-44.

Asghari, J., \& Musavi, S. Y. (2002). Allelopathic effects of rice varieties on barnyardgrass and umbrella sedge. Journal of Plant Diseases, 38, 133-143.

Chung, I. M., Ahn, J. K., \& Yun, S. J. (2001). Identification of allelopathic compounds from rice hull and their biological activity. J Plant Sci., 81, 815-819.

Chung, I. M., Kim, K. H., Ahn, J. K., Lee, S. B., Kim, S. H., \& Hahn, S. J. (2003). Comparison of allelopathic potential of Rice leaves, straw and Hall Extracts on Barnyard grass. Agron. J., 95, 1063-1040. http://dx.doi.org/10.2134/agronj2003.1063

Dilday, R. H., Lin, J., \& Yan, W. (1994). Identification of allelopathy in the USDA-ARS rice germplasm collection. Aust J Exp Agric, 34, 907-10. http://dx.doi.org/10.1071/EA9940907

Ferrero, A., \& Tinarelli, A. (2008). Pesticide Risk Assessment in Rice Paddies: Theory and Practice. Rice Cultivation in the E.U. Ecological Conditions and Agronomical Practices, Elsevier.

Hassan, S. M., Aidy, I. R., \& Bastawisi, A. O. (1995). Allelopathic potential of rice varieties against major weeds in Egypt. Paper presented at annual metting of weed science society of America, Seatlle, Washington, USA.

Hassan, S. M., Rao, A. N., Bastawisi, A. O., \& Aidy, I. R. (1994). Weed management in wet seeded in rice in Egypt. IRRI (pp. 257-269).

He, H., Wang, H., Ang, C., Wu, H., Guo, X., Liu, C., ... Lin, W. (2012). Barnyard grass stress up regulates the biosynthesis of phenolic compounds in allelopathic rice. Journal of Plant Physiology, 169, 1747-1753. http://dx.doi.org/10.1016/j.jplph.2012.06.018

Kim, K. U., \& Shin, D. H. (1996). Rice allelopathy research in Korea. Weed Sci., 10(3), 221-226.

Kim, S. Y., Madrid, A. V., Park, S. T., Yang, S. J., \& Olofsdotter, M. (2005). Evaluation of rice allelopathy in hydroponics. Weed Research, 45, 74-79. http://dx.doi.org/10.1111/j.1365-3180.2004.00438.x

Olofsdotter, M. (1998). Allelopathy in rice. In M. Olofsdotter (Ed.), Allelopathy in rice (pp. 1-5). Proceedings of the workshop on allelopatny in rice, International Rice Research Institute, Manila, Philippines.

Olofsdotter, M., \& Navare, D. (1996). Allelopatic rice for Echinochloa cruss-galli control (pp. 1175-1181). 2nd International weed control congress, Denmark.

SAS Institute Incorporation. (1997). SAS/STAT users Guide (6.03 ed., p. 108). SAS Institude.

Tang, J., Xu, L., Chen, X., \& Hu, S. (2009). Interaction between C4 barnyard grass and C3 upland rice under elevated CO2: Impact of mycorrhizae. Acta Eecologica, 35, 227-235. http://dx.doi.org/10.1016/j.actao.2008.10.005

Torres, A., Olive, R. M., Castella, D., \& Cross, P. (1996). First world congress on allelopathy. A science of the fure (p. 278). University of Candiz.

Xuan, T. D., Hong, N. H., Khan, T. D., Fiji, T., Tawata, S., \& Fukuta, M. (2006). Utilization of plant allelopathy for biological control of weeds and plant pathogens in rice. 13th Australian Agronomy conferences. 


\section{Copyrights}

Copyright for this article is retained by the author(s), with first publication rights granted to the journal.

This is an open-access article distributed under the terms and conditions of the Creative Commons Attribution license (http://creativecommons.org/licenses/by/3.0/). 